\title{
Article
}

\section{Heavy Metal Tolerance Trend in Extended-Spectrum $\beta$-Lactamase Encoding Strains Recovered from Food Samples}

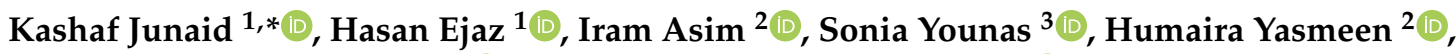 \\ Abualgasim Elgaili Abdalla ${ }^{1}$, Khalid Omer Abdalla Abosalif ${ }^{1}{ }^{\oplus}$, Ayman Ali Mohammed Alameen ${ }^{1}{ }^{\infty}$, \\ Naveed Ahmad ${ }^{4}\left(\mathbb{D}\right.$, Syed Nasir Abbas Bukhari ${ }^{5}\left(\mathbb{D}\right.$ and Abdul Rehman ${ }^{6}$
}

check for updates

Citation: Junaid, K.; Ejaz, H.; Asim, I.; Younas, S.; Yasmeen, H.; Abdalla, A.E.; Abosalif, K.O.A.; Alameen, A.A.M.; Ahmad, N.; Bukhari, S.N.A.; et al. Heavy Metal Tolerance Trend in Extended-Spectrum $\beta$-Lactamase Encoding Strains Recovered from Food Samples. Int. J. Environ. Res. Public Health 2021, 18, 4718. https:// doi.org/10.3390/ijerph18094718

\section{Academic Editors:}

Teresa Semedo-Lemsaddek and David Rodríguez-Lázaro

Received: 6 April 2021

Accepted: 27 April 2021

Published: 28 April 2021

Publisher's Note: MDPI stays neutral with regard to jurisdictional claims in published maps and institutional affiliations.

Copyright: (c) 2021 by the authors. Licensee MDPI, Basel, Switzerland. This article is an open access article distributed under the terms and conditions of the Creative Commons Attribution (CC BY) license (https:/ / creativecommons.org/licenses/by/ $4.0 /)$.
1 Department of Clinical Laboratory Sciences, College of Applied Medical Sciences, Jouf University, Skaka 72388, Saudi Arabia; hetariq@ju.edu.sa (H.E.); aealseddig@ju.edu.sa (A.E.A.); koabosalif@ju.edu.sa (K.O.A.A.); aaalameen@ju.edu.sa (A.A.M.A.)

2 Department of Microbiology and Molecular Genetics, The Women University, Multan 66000, Pakistan; Iramasim.mmg@gmail.com (I.A.); humaira.6127@wum.edu.pk (H.Y.)

3 Department of Pathology, Tehsil Headquarter Hospital Kamoke, Kamoke 50661, Pakistan; soniamicro02@gmail.com

4 Department of Pharmaceutics, College of Pharmacy, Jouf University, Sakaka 72388, Saudi Arabia; nakahmad@ju.edu.sa

5 Department of Pharmaceutical Chemistry, College of Pharmacy, Jouf University, Sakaka 72388, Saudi Arabia; sbukhari@ju.edu.sa

6 Department of Microbiology and Molecular Genetics, University of the Punjab, Lahore 54590, Pakistan; rehman_mmg@yahoo.com

* Correspondence: kjunaid@ju.edu.sa; Tel.: +966-55-237-8576

\begin{abstract}
This study evaluates bacteriological profiles in ready-to-eat (RTE) foods and assesses antibiotic resistance, extended-spectrum $\beta$-lactamase (ESBL) production by gram-negative bacteria, and heavy metal tolerance. In total, 436 retail food samples were collected and cultured. The isolates were screened for ESBL production and molecular detection of ESBL-encoding genes. Furthermore, all isolates were evaluated for heavy metal tolerance. From 352 culture-positive samples, 406 gnegative bacteria were identified. Raw food samples were more often contaminated than refined food $(84.71 \%$ vs. $76.32 \%)$. The predominant isolates were Klebsiella pneumoniae $(n=76)$, Enterobacter cloacae $(n=58)$, and Escherichia coli $(n=56)$. Overall, the percentage of ESBL producers was higher in raw food samples, although higher occurrences of ESBL-producing E. coli $(p=0.01)$ and Pseudomonas aeruginosa $(p=0.02)$ were observed in processed food samples. However, the prevalence of ESBLproducing Citrobacter freundii in raw food samples was high $(p=0.03)$. Among the isolates, 55\% were $b l a_{\mathrm{CTX}-\mathrm{M}}, 26 \%$ were $b l a_{\mathrm{SHV}}$, and $19 \%$ were $b l a_{\mathrm{TEM}}$. Notably, heavy metal resistance was highly prevalent in ESBL producers. These findings demonstrate that retail food samples are exposed to contaminants including antibiotics and heavy metals, endangering consumers.
\end{abstract}

Keywords: processed food; food contaminants; heavy metals; beta-lactamase; ESBL

\section{Introduction}

Microbiological risks associated with consuming ready-to-eat (RTE) food have recently become more common. Numerous outbreaks of pathogenic microorganisms demonstrate that food items are potential carriers of microorganisms that cause foodborne diseases [1]. RTE food contains pathogenic and nonpathogenic microbial flora, particularly gram-negative bacteria such as Escherichia, Vibrio, Shigella, Salmonella, Campylobacter, and Klebsiella species [2,3].

The incidence of foodborne diseases is high in developing countries. However, no region of the world is devoid of foodborne diseases [4]. Several studies have found extended-spectrum $\beta$-lactamase (ESBL)-producing organisms in fruits and vegetables in retail $[1,5]$. Recently, a study reported the prevalence of ESBL on bean sprouts and 
other vegetables in South Korea and the Netherlands [6]. Through the application of animal-product biofertilizers, edible plants and fruits may become infected with antibioticresistant and pathogenic bacteria during development and serve as vectors of transmission to humans [7].

Moreover, a significant association between food handlers and foodborne outbreaks of ESBL-producing Enterobacteriaceae was reported in Spain [8]. In another study, a nosocomial outbreak of ESBL-producing K. pneumoniae was also reported and the drug resistant strain was transmitted through food [9]. Additionally, a study in Kenya determined that fecal ESBL contaminants in food (3\%) originated from food handlers [10].

The accessibility of inexpensive RTE food comes at the cost of quality in low-income populations [11]. Unhygienic handling of food from production through delivery is often seen in Pakistan. There are many opportunities for multi-resistant foodborne pathogen contamination of retail food items.

The association between heavy metal-tolerant bacteria in food items and antibacterial drug resistance is insufficiently studied. Antibiotic-resistant bacteria are enriched in metal-contaminated environments, and genes conferring co-selection for heavy metal- and antibiotic-tolerance are frequently found together in clinical isolates [12,13]. The association between antibiotic-resistant bacteria and raw and processed foods is also poorly understood. This study evaluated the prevalence of ESBL-producing gram-negative isolates in retail food samples. In addition, isolates were compared to determine the association between ESBL production and heavy metal tolerance.

\section{Materials and Methods}

\subsection{Study Design and Sample Collection}

In this study, 436 different food samples were collected from seven Punjab districts (Bahawalpur, Jahania, Multan, Budhla Sant, Dunyapur, Jhang, and Khanewal) in Pakistan. All food samples were collected from street vendors, local markets, hospitals, and university cafeterias. In total, 207 processed food samples (fast food and cooked meat, chicken, fish, rice, and desserts) and 229 raw or unprocessed food samples (raw vegetables/fruits, salads, juices, and milkshakes) were collected. The details of all included samples and the sample flow are presented in Figure 1. The samples were aseptically collected, i.e., solid food was placed in sterile bags, and liquid food was collected in sterile screw-capped bottles. All samples were kept at $4{ }^{\circ} \mathrm{C}$ until further processing.

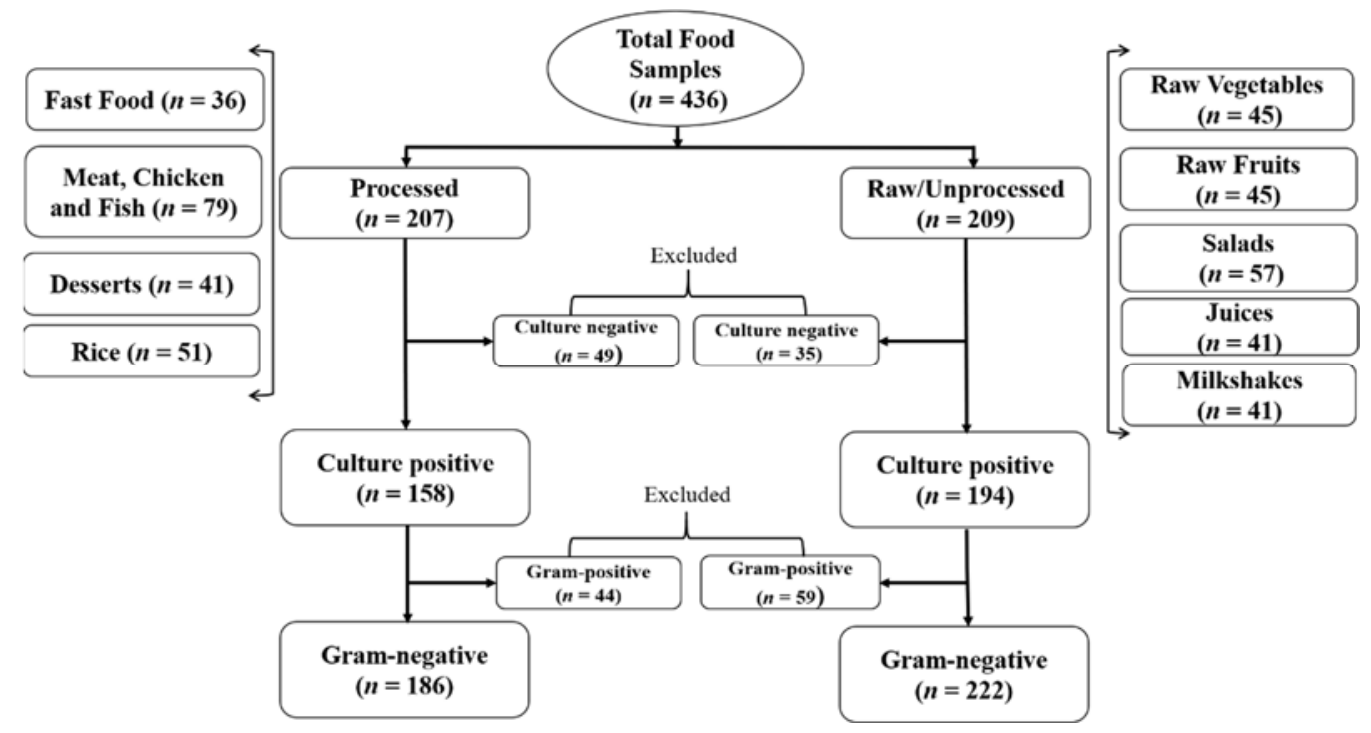

Figure 1. Types of food samples collected and processed in this study $(n=436)$. 


\subsection{Specimen Collection and Processing}

For processing, $250 \mathrm{~g}$ of solid food samples and $10 \mathrm{~mL}$ of liquid samples were collected for microbiological analysis. Solid samples were enriched by crushing with a mortar and pestle. Subsequently, $25 \mathrm{~g}$ of the crushed specimen was homogenized with $225 \mathrm{~mL}$ of peptone water broth. The homogenized mixture was shaken $(160 \mathrm{rpm})$ for $1-2 \mathrm{~h}$ and then further processed.

\subsection{Isolation and Characterization of Gram-Negative Bacteria}

Enriched cultures and liquid samples were streaked onto MacConkey agar, and the plates were incubated at $37{ }^{\circ} \mathrm{C}$ overnight. Presumptive identification of isolates was performed based on colony morphology, Gram staining, and biochemical tests $[14,15]$. Only gram-negative isolates were further subjected to biochemical identification. For additional confirmation, the API 20E and 20NE tests were performed.

\subsection{Antimicrobial Sensitivity Testing}

In vitro antimicrobial drug sensitivity was evaluated using 18 drugs recommended for use against gram-negative bacteria. Each isolate's colonies were aseptically emulsified in normal saline to produce a suspension meeting to $0.5 \mathrm{McF}$ arland standards. The suspended isolate was streaked onto a Mueller Hinton agar culture plate, and antibiotic discs were placed on the culture plate surface. Culture plates were incubated at $37^{\circ} \mathrm{C}$ for $18-24 \mathrm{~h}$, and each organism was reported as sensitive or resistant to each antibiotic according to the zone of inhibition. The following antibiotics were used in this study: aztreonam $(30 \mu \mathrm{g})$, amikacin $(30 \mu \mathrm{g})$, gentamicin $(10 \mu \mathrm{g})$, cefuroxime $(30 \mu \mathrm{g})$, cefoxitin $(30 \mu \mathrm{g})$, ceftriaxone $(30 \mu \mathrm{g})$, ceftazidime $(30 \mu \mathrm{g})$, cefotaxime $(30 \mu \mathrm{g})$, cefepime $(30 \mu \mathrm{g})$, ciprofloxacin $(5 \mu \mathrm{g})$, levofloxacin $(5 \mu \mathrm{g})$, imipenem $(10 \mu \mathrm{g})$, meropenem $(10 \mu \mathrm{g})$, piperacillin-tazobactam $(100 / 10 \mu \mathrm{g})$, colistin $(10 \mu \mathrm{g})$, co-trimoxazole $(1.25 / 23.75 \mu \mathrm{g})$, and tigecycline $(15 \mu \mathrm{g})$. To ensure the reliability of in vitro antibacterial sensitivity testing, ATCC quality control (QC) strains of K. pneumoniae (700603, ESBL-positive) and E. coli (25922, ESBL-negative) were used, as recommended by CLSI guidelines [16].

\subsection{Phenotypic Characterization of ESBL Producers}

According to accepted guidelines, the double-disc synergy test and the combineddisc test were performed on Mueller Hinton agar plates to characterize the phenotypic characteristics of ESBL-producing strains. Cephalosporin and clavulanate antibiotics were used in both tests [17].

\subsection{Molecular Characterization of MCR Genes}

Genes encoding ESBL were amplified from all phenotypically characterized ESBL producers. Previously reported primers for $b l a_{\mathrm{SHV}}, b l a_{\mathrm{TEM}}$, and $b l a_{\mathrm{CTX}-\mathrm{M}}$ were used, and the PCR conditions were optimized [18]. PCR conditions were followed as: initial denaturation at $95^{\circ} \mathrm{C}$ for $3 \mathrm{~min}$; 30 cycles of $15 \mathrm{~s}$ at $95{ }^{\circ} \mathrm{C}, 56{ }^{\circ} \mathrm{C}$ for $35 \mathrm{~s}$ and $72{ }^{\circ} \mathrm{C}$ for $1 \mathrm{~min}$; finally, a 7 min extension step at $72{ }^{\circ} \mathrm{C}$. The amplified products were visualized by agarose gel electrophoresis, and product sizes were determined using a DNA ladder (100 bp). A UV transilluminator and gel documentation system were used to collect digital images of the PCR products. The PCR products were submitted to reverse and forward gene sequencing and analyzed using FinchTV v. 1.4 (Geospiza, Inc., Seattle, WA, USA). To identify bla gene variants, programs such as BlastN, BlastP (NCBI), and ExPASy (SIB Group) were used for nucleotide, amino acid, and translational analyses.

\subsection{Resistance to Heavy Metals}

According to a previously published procedure, the heavy metal tolerance of each isolate was examined $[19,20]$ using the heavy metal salts molybdenum oxide, chromium chloride, arsenic chloride, and cobalt chloride. Briefly, each isolate was assessed on agar plates containing increasing concentrations of each heavy metal salt. The heavy metal 
concentration ranged between $250 \mu \mathrm{g} / 100 \mathrm{~mL}$ and $1500 \mu \mathrm{g} / 100 \mathrm{~mL}$. Each plate with a higher heavy metal concentration was inoculated with the isolate from the plate with the previous concentration, and the plates were incubated at $37{ }^{\circ} \mathrm{C}$ for $24 \mathrm{~h}$ and examined for growth.

\subsection{Statistical Analysis}

The data were analyzed using SPSS version 24.0 (Armonk, NY, USA) and GraphPad Prism 6.0 (San Diego, CA, USA). Descriptive analysis was performed to calculate the frequencies and percentages of each variable. The chi-square test and regression analysis were used for inferential statistics.

\section{Results}

The results revealed that of 436 food samples collected in this study, 352 (80.73\%) were culture-positive. Raw food samples were more often contaminated than processed food samples $(84.71 \%$ vs. $76.32 \%)$. The average CFU count for raw food samples was $7.4 \times 10^{5}$, and $5.6 \times 10^{4}$ for processed food samples was observed. From the 352 culture-positive samples, 408 g-negative bacterial isolates (186 from processed food samples and 222 from raw food samples) were obtained and identified. Among the isolates, the most abundant organisms were K. pneumoniae $(n=76)$, E. cloacae $(n=58)$, E. coli $(n=56)$, S. typhimurium $(n=43)$, Serratia marcescens $(n=38)$, S. enteritidis $(n=35), C$. freundii $(n=29)$, Y. enterocolitica $(n=21)$, P. aeruginosa $(n=19)$, Shigella spp. $(n=17)$, and P. mirabilis $(n=16)$.

\subsection{Association of Isolates with the Food Sample Type}

The most prevalent isolate from the raw food sample was K. pneumoniae (19.82\%), and from processed food samples, it was E. coli $(20.43 \%)$. E. coli $(20.43 \%, p<0.01)$ and $P$. aeruginosa $(7.53 \%, p=0.01)$ were associated with processed food. In contrast, $S$. typhimurium $(14.4 \%, p=0.01)$ and $S$. marcescens $(12.16 \%, p=0.03)$ were potentially associated with raw food. No marked differences in prevalence according to the food sample type were observed for the other organisms (Table 1).

Table 1. Gram-negative isolates from processed and raw food samples $(n=408)$.

\begin{tabular}{cccc}
\hline Isolates $(\boldsymbol{n} ; \mathbf{\%})$ & $\begin{array}{c}\text { Processed Food } \\
\text { Samples }(\boldsymbol{n}=\mathbf{1 8 6})\end{array}$ & $\begin{array}{c}\text { Raw Food Samples } \\
(\boldsymbol{n}=\mathbf{2 2 2})\end{array}$ & $\boldsymbol{p}$-Value \\
\hline E. coli $(56 ; 13.7 \%)$ & $38(20.43 \%)$ & $18(8.11 \%)$ & $<0.01$ \\
K. pneumoniae $(76 ; 18.6 \%)$ & $32(17.20 \%)$ & $44(19.82 \%)$ & 0.49 \\
E. cloacae $(58 ; 14.2 \%)$ & $22(11.83 \%)$ & $36(16.22 \%)$ & 0.20 \\
C. freundii $(29 ; 7.1 \%)$ & $16(8.60 \%)$ & $13(5.86 \%)$ & 0.28 \\
P. aeruginosa $(19 ; 4.7 \%)$ & $14(7.53 \%)$ & $5(2.25 \%)$ & 0.01 \\
S. enteritidis $(35 ; 8.6 \%)$ & $14(7.53 \%)$ & $21(9.46 \%)$ & 0.48 \\
Y. enterocolitica $(21 ; 5.1 \%)$ & $13(6.99 \%)$ & $8(3.60 \%)$ & 0.12 \\
S. typhimurium $(43 ; 10.5 \%)$ & $11(5.91 \%)$ & $32(14.41 \%)$ & 0.01 \\
S. marcescens $(38 ; 9.3 \%)$ & $11(5.91 \%)$ & $27(12.16 \%)$ & 0.03 \\
Shigella spp. $(17 ; 4.2 \%)$ & $9(4.84 \%)$ & $8(3.60 \%)$ & 0.53 \\
P. mirabilis $(16 ; 3.9 \%)$ & $6(3.23 \%)$ & $10(4.50 \%)$ & 0.50 \\
\hline
\end{tabular}

p-values were obtained from the chi-square test.

\subsection{Antimicrobial Sensitivity Pattern}

The overall results of antibacterial drug resistance testing against 18 drugs showed that most isolates were resistant to ampicillin (90\%). The highest cephalosporin antibiotic resistance was observed for cefuroxime (51\%), and the lowest resistance was observed for cefoxitin and cefepime (22\%). The carbapenem and quinolone resistance percentages were similar $(17 \%$ and $18 \%$, respectively). This study revealed minimal resistance to tigecycline $(6 \%)$ and colistin $(2 \%)$. The detailed antibacterial drug resistance profiles of the individual bacterial species are presented in Table 2. 
Table 2. Antimicrobial resistance profiles of food isolates $(n=408)$.

\begin{tabular}{|c|c|c|c|c|c|c|c|c|c|c|c|}
\hline Antibiotics & 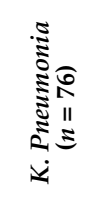 & 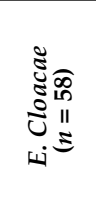 & $\begin{array}{l}: 00 \\
011 \\
ن=3\end{array}$ & 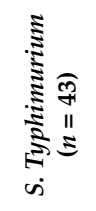 & 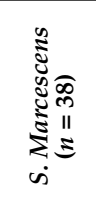 & 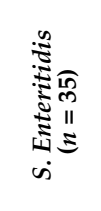 & 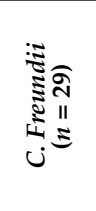 & 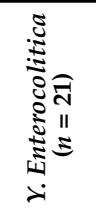 & 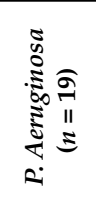 & 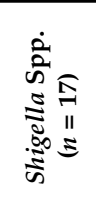 & 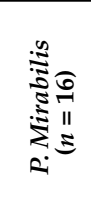 \\
\hline Ampicillin & IR & IR & $\begin{array}{c}41 \\
73.21 \%\end{array}$ & $\begin{array}{c}32 \\
74.42 \%\end{array}$ & IR & $\begin{array}{c}26 \\
74.29 \%\end{array}$ & IR & IR & IR & $\begin{array}{c}9 \\
52.94 \%\end{array}$ & $\begin{array}{c}11 \\
68.75 \%\end{array}$ \\
\hline Aztreonam & $\begin{array}{c}19 \\
25 \%\end{array}$ & $\begin{array}{c}23 \\
39.66 \%\end{array}$ & $\begin{array}{c}16 \\
28.57 \%\end{array}$ & $\begin{array}{c}15 \\
34.88 \%\end{array}$ & $\begin{array}{c}12 \\
31.58 \%\end{array}$ & $\begin{array}{c}21 \\
60 \%\end{array}$ & $\begin{array}{c}9 \\
31.03 \%\end{array}$ & $\begin{array}{c}10 \\
47.62 \%\end{array}$ & $\begin{array}{c}8 \\
42.11 \%\end{array}$ & $\begin{array}{c}4 \\
23.53 \%\end{array}$ & $\begin{array}{c}6 \\
37.5 \%\end{array}$ \\
\hline Amikacin & $\begin{array}{c}24 \\
31.58 \%\end{array}$ & $\begin{array}{c}26 \\
44.83 \%\end{array}$ & $\begin{array}{c}19 \\
33.93 \%\end{array}$ & $\begin{array}{c}13 \\
30.23 \%\end{array}$ & $\begin{array}{c}19 \\
50 \%\end{array}$ & $\begin{array}{c}27 \\
77.14 \%\end{array}$ & $\begin{array}{c}9 \\
31.03 \%\end{array}$ & $\begin{array}{c}9 \\
42.86 \%\end{array}$ & $\begin{array}{c}8 \\
42.11 \%\end{array}$ & $\begin{array}{c}4 \\
23.53 \%\end{array}$ & $\begin{array}{c}7 \\
43.75 \%\end{array}$ \\
\hline Gentamicin & $\begin{array}{c}21 \\
27.63 \%\end{array}$ & $\begin{array}{c}24 \\
41.38 \%\end{array}$ & $\begin{array}{c}16 \\
28.57 \%\end{array}$ & $\begin{array}{c}12 \\
27.91 \%\end{array}$ & $\begin{array}{c}19 \\
50 \%\end{array}$ & $\begin{array}{c}27 \\
77.14 \%\end{array}$ & $31.03 \%$ & $\begin{array}{c}8 \\
38.10 \%\end{array}$ & $\begin{array}{c}7 \\
36.84 \%\end{array}$ & $\begin{array}{c}4 \\
23.53 \%\end{array}$ & $\begin{array}{c}7 \\
43.75 \%\end{array}$ \\
\hline Cefuroxime & $\begin{array}{c}28 \\
36.84 \%\end{array}$ & $\begin{array}{c}29 \\
50 \%\end{array}$ & $\begin{array}{c}19 \\
33.93 \%\end{array}$ & $\begin{array}{c}15 \\
34.88 \%\end{array}$ & IR & $\begin{array}{c}13 \\
37.14 \%\end{array}$ & IR & $\begin{array}{c}11 \\
52.38 \%\end{array}$ & $\begin{array}{c}9 \\
47.37 \%\end{array}$ & $\begin{array}{c}7 \\
41.18 \%\end{array}$ & $\begin{array}{c}8 \\
50 \%\end{array}$ \\
\hline Cefoxitin & $\begin{array}{c}19 \\
25 \%\end{array}$ & IR & $\begin{array}{c}17 \\
30.36 \%\end{array}$ & $\begin{array}{c}19 \\
44.19 \%\end{array}$ & IR & $\begin{array}{c}12 \\
34.29 \%\end{array}$ & IR & $\begin{array}{c}9 \\
42.86 \%\end{array}$ & $\begin{array}{c}8 \\
42.11 \%\end{array}$ & $\begin{array}{c}2 \\
11.76 \%\end{array}$ & $\begin{array}{c}1 \\
6.25 \%\end{array}$ \\
\hline Ceftriaxone & $\begin{array}{c}21 \\
27.63 \%\end{array}$ & $\begin{array}{c}34 \\
58.62 \%\end{array}$ & $\begin{array}{c}19 \\
33.93 \%\end{array}$ & $\begin{array}{c}15 \\
34.88 \%\end{array}$ & $\begin{array}{c}19 \\
50 \%\end{array}$ & $\begin{array}{c}21 \\
60 \%\end{array}$ & $\begin{array}{c}9 \\
31.03 \%\end{array}$ & $\begin{array}{c}11 \\
52.38 \%\end{array}$ & IR & $\begin{array}{c}7 \\
41.18 \%\end{array}$ & $\begin{array}{c}3 \\
18.75 \%\end{array}$ \\
\hline Ceftazidime & $\begin{array}{c}23 \\
30.26 \%\end{array}$ & $\begin{array}{c}35 \\
60.34 \%\end{array}$ & $\begin{array}{c}21 \\
37.50 \%\end{array}$ & $\begin{array}{c}17 \\
39.53 \%\end{array}$ & $\begin{array}{c}19 \\
50 \%\end{array}$ & $\begin{array}{c}27 \\
77.14 \%\end{array}$ & $\begin{array}{c}9 \\
31.03 \%\end{array}$ & $\begin{array}{c}11 \\
52.38 \%\end{array}$ & $\begin{array}{c}8 \\
42.11 \%\end{array}$ & $\begin{array}{c}7 \\
41.18 \%\end{array}$ & $\begin{array}{c}7 \\
43.75 \%\end{array}$ \\
\hline Cefotaxime & $\begin{array}{c}24 \\
31.58 \%\end{array}$ & $\begin{array}{c}32 \\
55.17 \%\end{array}$ & $\begin{array}{c}25 \\
44.64 \%\end{array}$ & $\begin{array}{c}26 \\
60.47 \%\end{array}$ & $\begin{array}{c}17 \\
44.74 \%\end{array}$ & $\begin{array}{c}18 \\
51.43 \%\end{array}$ & $\begin{array}{c}17 \\
58.62 \%\end{array}$ & $\begin{array}{c}11 \\
52.38 \%\end{array}$ & IR & $\begin{array}{c}9 \\
52.94 \%\end{array}$ & $\begin{array}{c}8 \\
50 \%\end{array}$ \\
\hline Cefepime & $\begin{array}{c}17 \\
22.37 \%\end{array}$ & $\begin{array}{c}7 \\
12.07 \%\end{array}$ & $\begin{array}{c}16 \\
28.57 \%\end{array}$ & $\begin{array}{c}6 \\
13.95 \%\end{array}$ & $\begin{array}{c}4 \\
10.53 \%\end{array}$ & $\begin{array}{c}16 \\
45.71 \%\end{array}$ & $\begin{array}{c}3 \\
10.34 \%\end{array}$ & $\begin{array}{c}7 \\
33.33 \%\end{array}$ & $\begin{array}{c}2 \\
10.53 \%\end{array}$ & $23.53 \%$ & $\begin{array}{c}5 \\
31.25 \%\end{array}$ \\
\hline Ciprofloxacin & $\begin{array}{c}17 \\
22.37 \%\end{array}$ & $\begin{array}{c}9 \\
15.52 \%\end{array}$ & $\begin{array}{c}11 \\
19.64 \%\end{array}$ & $\begin{array}{c}7 \\
16.28 \%\end{array}$ & $\begin{array}{c}4 \\
10.53 \%\end{array}$ & $\begin{array}{c}7 \\
20 \%\end{array}$ & $\begin{array}{c}3 \\
10.34 \%\end{array}$ & $\begin{array}{c}5 \\
23.81 \%\end{array}$ & $\begin{array}{c}2 \\
10.53 \%\end{array}$ & $\begin{array}{c}5 \\
29.41 \%\end{array}$ & $\begin{array}{c}3 \\
18.75 \%\end{array}$ \\
\hline Levofloxacin & $\begin{array}{c}19 \\
25 \%\end{array}$ & $\begin{array}{c}12 \\
20.69 \%\end{array}$ & $\begin{array}{c}10 \\
17.86 \%\end{array}$ & $\begin{array}{c}7 \\
16.28 \%\end{array}$ & $\begin{array}{c}3 \\
7.89 \%\end{array}$ & $\begin{array}{c}6 \\
17.14 \%\end{array}$ & $\begin{array}{c}3 \\
10.34 \%\end{array}$ & $\begin{array}{c}4 \\
19.05 \%\end{array}$ & $\begin{array}{c}2 \\
10.53 \%\end{array}$ & $\begin{array}{c}5 \\
29.41 \%\end{array}$ & $\begin{array}{c}3 \\
18.75\end{array}$ \\
\hline Imipenem & $\begin{array}{c}17 \\
22.37 \%\end{array}$ & $\begin{array}{c}6 \\
10.34 \%\end{array}$ & $\begin{array}{c}13 \\
23.21 \%\end{array}$ & $\begin{array}{c}6 \\
13.95 \%\end{array}$ & $\begin{array}{c}4 \\
10.53 \%\end{array}$ & $\begin{array}{c}7 \\
20 \%\end{array}$ & $\begin{array}{c}3 \\
10.34 \%\end{array}$ & $\begin{array}{c}5 \\
23.81 \%\end{array}$ & $\begin{array}{c}2 \\
10.53 \%\end{array}$ & $\begin{array}{c}3 \\
17.65 \%\end{array}$ & $\begin{array}{c}3 \\
18.75 \%\end{array}$ \\
\hline Meropenem & $\begin{array}{c}1 \\
23.68 \%\end{array}$ & $\begin{array}{c}6 \\
10.34 \%\end{array}$ & $\begin{array}{c}12 \\
21.43 \%\end{array}$ & $\begin{array}{c}6 \\
13.95\end{array}$ & $\begin{array}{c}4 \\
10.53 \%\end{array}$ & $\begin{array}{c}8 \\
22.86 \%\end{array}$ & $\begin{array}{c}3 \\
10.34 \%\end{array}$ & $\begin{array}{c}5 \\
23.81 \%\end{array}$ & $\begin{array}{c}2 \\
10.53 \%\end{array}$ & $\begin{array}{c}3 \\
17.65 \%\end{array}$ & $\begin{array}{c}3 \\
18.75 \%\end{array}$ \\
\hline Piperacillin-Tazobactam & $\begin{array}{c}1 \\
15.79 \%\end{array}$ & $\begin{array}{c}6 \\
10.34 \%\end{array}$ & $\begin{array}{c}5 \\
8.93 \%\end{array}$ & $\begin{array}{c}6 \\
13.95 \%\end{array}$ & $\begin{array}{c}4 \\
10.53 \%\end{array}$ & $22.86 \%$ & $\begin{array}{c}3 \\
10.34 \%\end{array}$ & $\begin{array}{c}4 \\
19.05 \%\end{array}$ & $\begin{array}{c}2 \\
10.53 \%\end{array}$ & $\begin{array}{c}3 \\
17.65 \%\end{array}$ & $\begin{array}{c}3 \\
18.75 \%\end{array}$ \\
\hline Colistin & $\begin{array}{c}1 \\
1.32 \%\end{array}$ & $1.72 \%$ & $\stackrel{2}{2}$ & $\begin{array}{c}1 \\
2.33 \%\end{array}$ & IR & $\begin{array}{c}1 \\
2.86 \%\end{array}$ & $\begin{array}{c}0 \\
0.00\end{array}$ & $\begin{array}{c}1 \\
4.76 \%\end{array}$ & $\begin{array}{c}0 \\
0 \%\end{array}$ & $\begin{array}{c}0 \\
0 \%\end{array}$ & IR \\
\hline Co-trimoxazole & $\begin{array}{c}22 \\
28.95 \%\end{array}$ & $\begin{array}{c}21 \\
36.21 \%\end{array}$ & $\begin{array}{c}16 \\
28.57 \%\end{array}$ & $\begin{array}{c}15 \\
34.88 \%\end{array}$ & $\begin{array}{c}12 \\
31.58 \%\end{array}$ & $\begin{array}{c}14 \\
40 \%\end{array}$ & $\begin{array}{c}9 \\
31.03 \%\end{array}$ & $\begin{array}{c}10 \\
47.62 \%\end{array}$ & $\begin{array}{c}8 \\
42.11 \%\end{array}$ & $\begin{array}{c}4 \\
23.53 \%\end{array}$ & $\begin{array}{c}6 \\
37.5 \%\end{array}$ \\
\hline Tigecycline & $\begin{array}{c}7 \\
9.21 \%\end{array}$ & $\begin{array}{c}3 \\
5.17 \%\end{array}$ & $\begin{array}{c}2 \\
3.57 \%\end{array}$ & $\begin{array}{c}6 \\
13.95 \%\end{array}$ & $\begin{array}{c}2 \\
5.26 \%\end{array}$ & $\begin{array}{c}1 \\
2.86 \%\end{array}$ & $\begin{array}{c}1 \\
3.45 \%\end{array}$ & $\begin{array}{c}1 \\
4.76 \%\end{array}$ & IR & $\begin{array}{c}1 \\
5.88 \%\end{array}$ & IR \\
\hline
\end{tabular}

IR: intrinsic resistance.

\subsection{Detection of ESBL-Producing Organisms and Associations with Food Samples}

Of the 408 isolates from food samples, 173 (42.4\%) exhibited ESBL production, including $84(48.5 \%)$ from processed food samples and 89 (51.5\%) from raw food samples. The following isolates with resistant phenotypes were identified: K. pneumoniae $(n=39)$, E. cloacae $(n=16)$, E. coli $(n=36)$, S. typhimurium $(n=17)$, Serratia marcescens $(n=7), S$. enteritidis $(n=11), C$. freundii $(n=14), Y$. enterocolitica $(n=5)$, P. aeruginosa $(n=14)$, Shigella spp. $(n=4)$, and P. mirabilis $(n=10)$.

We assessed the presence of ESBL-producing organisms relative to the food type. The results indicated a higher prevalence of ESBL-producing E. coli ( $p=0.01$, odds ratio (OR): 2.64 and $95 \%$ confidence interval (CI): $1.22-5.72)$ and P. aeruginosa ( $p=0.02$, OR: 4.43 and 95\% CI: 1.19-12.60) in processed food samples. In raw food samples, the incidence of ESBL-producing C. freundii was high ( $p=0.03$, OR: 3.71 and 95\% CI: 1.52-12.50). However, the other ESBL-producing organisms did not show any statistically significant associations with the food sample type in this study (Table 3). 
Table 3. Gram-negative isolates from processed and raw food samples $(n=408)$.

\begin{tabular}{ccccc}
\hline Isolates $(\boldsymbol{n} ; \mathbf{\%})$ & $\begin{array}{c}\text { Processed } \\
\text { Food }(\boldsymbol{n = 8 4 )}\end{array}$ & $\begin{array}{c}\text { Raw Food } \\
(\boldsymbol{n}=\mathbf{8 9 )}\end{array}$ & $\boldsymbol{p}$-Value & OR (95\% CI) \\
\hline K. pneumoniae $(39 ; 22.54 \%)$ & $17(43.59 \%)$ & $22(56.41 \%)$ & 0.53 & $0.8(0.39-1.63)$ \\
E. cloacae $(16 ; 9.25 \%)$ & $6(37.50 \%)$ & $10(62.50 \%)$ & 0.37 & $0.62(0.22-1.8)$ \\
E. coli $(36 ; 20.81 \%)$ & $24(66.67 \%)$ & $12(33.33 \%)$ & 0.01 & $2.64(1.22-5.72)$ \\
S. typhimurium $(17 ; 9.83 \%)$ & $8(47.06 \%)$ & $9(52.94 \%)$ & 0.93 & $0.96(0.35-2.62)$ \\
S. marcescens $(7 ; 4 \%)$ & $2(28.57 \%)$ & $5(71.43 \%)$ & 0.29 & $0.42(0.08-2.22)$ \\
S. enteritidis $(11 ; 6.36 \%)$ & $5(45.45 \%)$ & $6(54.55 \%)$ & 0.86 & $0.9(0.26-3.06)$ \\
C. freundii $(14 ; 8.1 \%)$ & $3(21.43 \%)$ & $11(78.57 \%)$ & 0.03 & $3.71(1.52-12.50)$ \\
Y. enterocolitica $(5 ; 2.89 \%)$ & $2(40 \%)$ & $3(60 \%)$ & 0.69 & $0.72(0.12-4.1)$ \\
P. aeruginosa $(14 ; 8.1 \%)$ & $11(78.57 \%)$ & $3(21.43 \%)$ & 0.02 & $4.43(1.19-12.60)$ \\
Shigella spp. $(4 ; 2.31 \%)$ & $3(75 \%)$ & $1(25 \%)$ & 0.273 & $3.34(0.34-31.74)$ \\
P. mirabilis $(10 ; 5.78 \%)$ & $3(30 \%)$ & $7(70 \%)$ & 0.24 & $0.44(0.11-1.78)$ \\
\hline
\end{tabular}

OR: odds ratio; CI: confidence interval; $p$-values were obtained from the chi-square test, and odds ratios were obtained by regression analysis.

\subsection{Molecular Characterization of ESBL-Encoding Genes}

Of 173 isolates phenotypically characterized as ESBL-producing, $150(86.71 \%)$ harbored ESBL-encoding genes. According to PCR results, $b l a_{\mathrm{CTX}-\mathrm{M}}, b l a_{\mathrm{SHV}}$, and $b l a_{\mathrm{TEM}}$ genes were detected in $83(55 \%), 39(26 \%)$, and $28(19 \%)$ isolates, respectively. Among K. pneumoniae isolates, $61 \%, 25 \%$, and $14 \%$ were positive for $b l a_{\mathrm{CTX}-\mathrm{M}}, b l a_{\mathrm{TEM}}$, and $b l a_{\mathrm{SHV}}$, respectively. Among E. cloacae isolates, $58 \%, 28 \%$, and $14 \%$ harbored $b l a_{\mathrm{CTX}-\mathrm{M}}, b l a_{\mathrm{TEM}}$, and bla $a_{\mathrm{SHV}}$, re-

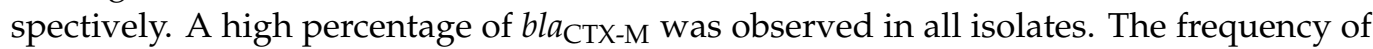
bla $_{\text {TEM }}$ was similar in E. coli, S. marcescens, and S. enteritidis. Similarly, the frequency of bla $a_{\mathrm{SHV}}$ was the same in S. enteritidis, Shigella spp., Y. enterocolitica, and P. aeruginosa. The distribution of ESBL-encoding genes in each isolate is presented in Figure 2.

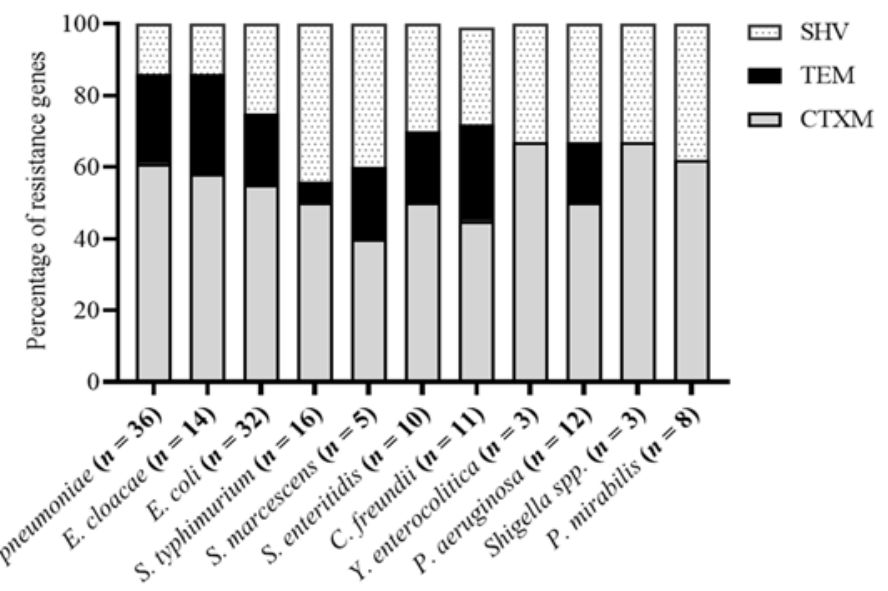

Figure 2. Genetic distribution of ESBL-encoding genes in the isolates $(n=150)$.

Moreover, the detailed distributions of gene variants in these isolates are presented in Table 4 . The highest incidence was reported for $b l a_{\mathrm{CTXM}-1}$ (56), followed by $b a_{\mathrm{SHV}-12}$ (35), $b l a_{\mathrm{TEM}-1}(25), b l a_{\mathrm{CTXM}-2}(21), b l a_{\mathrm{CTXM}-9}(6)$, and $b l a_{\mathrm{TEM}-135}$ (2), with only one isolate harboring $b l a_{\mathrm{TEM}-4}$. 
Table 4. Distributions of ESBL bla gene variants $(n=150)$.

\begin{tabular}{|c|c|c|c|c|c|c|c|c|c|c|}
\hline $\begin{array}{c}\text { Bla Gene Variants } \\
(n, \%)\end{array}$ & 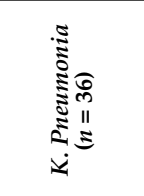 & 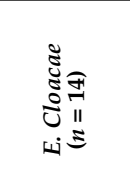 & 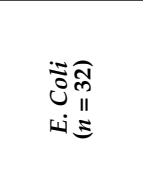 & 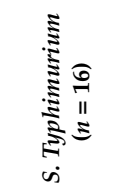 & 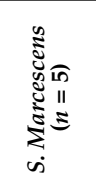 & 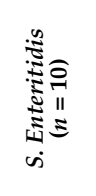 & 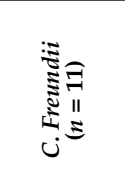 & 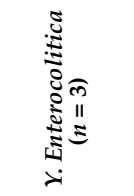 & 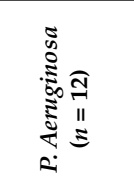 & 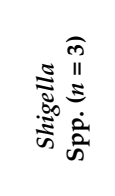 \\
\hline CTX-M-1 (56, 37.33\%) & $17(47.22 \%)$ & $5(35.71 \%)$ & $11(34.38 \%)$ & $6(37.5 \%)$ & $1(20 \%)$ & $3(30 \%)$ & $3(27.27 \%)$ & $2(66.67 \%)$ & $4(33.33 \%)$ & $1(33.33 \%)$ \\
\hline CTX-M-2 $(21,14 \%)$ & $3(8.33 \%)$ & $2(14.29 \%)$ & $6(18.75 \%)$ & $2(12.5 \%)$ & $1(20 \%)$ & $1(10 \%)$ & $2(18.18 \%)$ & $0(0 \%)$ & $2(16.67 \%)$ & $1(33.33 \%)$ \\
\hline CTX-M-9 $(6,4 \%)$ & $2(5.56 \%)$ & $1(7.14 \%)$ & $1(3.13 \%)$ & $0(0 \%)$ & $0(0 \%)$ & $1(10 \%)$ & $0(0 \%)$ & $0(0 \%)$ & $0(0 \%)$ & $0(0 \%)$ \\
\hline TEM-1 $(25,16.67 \%)$ & $8(22.22 \%)$ & $3(21.43 \%)$ & $6(18.75 \%)$ & $1(6.25 \%)$ & $1(20 \%)$ & $2(20 \%)$ & $2(18.18 \%)$ & $0(0 \%)$ & $2(16.67 \%)$ & $0(0 \%)$ \\
\hline TEM-135 $(2,1.33 \%)$ & $1(2.78 \%)$ & $0(0 \%)$ & $0(0 \%)$ & $0(0 \%)$ & $0(0 \%)$ & $0(0 \%)$ & $1(9.09 \%)$ & $0(0 \%)$ & $0(0 \%)$ & $0(0 \%)$ \\
\hline TEM-4 $(1,0.67 \%)$ & $0(0 \%)$ & $1(7.14 \%)$ & $0(0 \%)$ & $0(0 \%)$ & $0(0 \%)$ & $0(0 \%)$ & $0(0 \%)$ & $0(0 \%)$ & $0(0 \%)$ & $0(0 \%)$ \\
\hline SHV-12 $(35,23.33 \%)$ & $5(13.89 \%)$ & $2(14.29 \%)$ & $7(21.88 \%)$ & $5(31.25 \%)$ & $2(40 \%)$ & $3(30 \%)$ & $3(27.27 \%)$ & $1(33.33 \%)$ & $3(25 \%)$ & $1(33.33 \%)$ \\
\hline SHV-11 $(4,2.67 \%)$ & $0(0 \%)$ & $0(0 \%)$ & $1(3.13 \%)$ & $2(12.5 \%)$ & $0(0 \%)$ & $0(0 \%)$ & $0(0 \%)$ & $0(0 \%)$ & $1(8.33 \%)$ & $0(0 \%)$ \\
\hline
\end{tabular}

\subsection{Heavy Metal Resistance}

Further, all isolates were assessed for heavy metal resistance against four metals at increasing concentrations up to $1500 \mu \mathrm{g} / \mathrm{mL}$. The results indicated potential heavy metal contamination in the RTE food samples. No clear differences were observed among heavy metal resistance patterns. However, a uniform decrease in the number of resistant isolates was observed with increasing heavy metal concentrations. Selective screening of heavy metal-resistant isolates showed that the isolates with maximal resistance were also ESBL producers. Figure 3 presents the numbers of bacterial isolates in the ESBL and non-ESBL groups that showed resistance against heavy metals at the indicated concentrations.
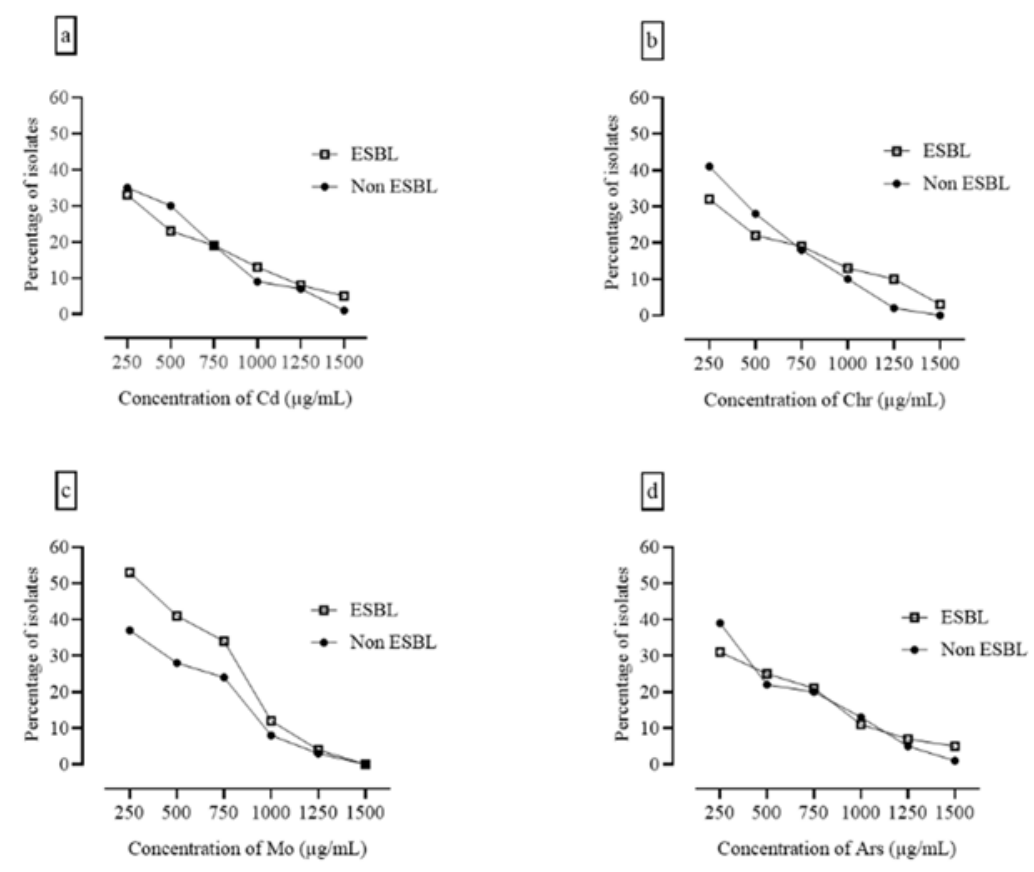

Figure 3. Number of heavy metal resistant isolates at different concentrations of heavy metals. As the concentration of heavy metal increased, the number of organisms decreased. (a) High resistance trend of ESBL producers against Cadmium. (b) High resistance trend of ESBL producers against Chromium. (c) High resistance trend of ESBL producers against Molybdinieum (d) High resistance trend of ESBL producers against Arsenic.

\section{Discussion}

Beta-lactamase-harboring gram-negative bacteria are no longer exclusively linked to the health care system. Therefore, investigating the possible threats to food safety and integrity posed by these bacteria has become increasingly important. The dissemination and expansion of antimicrobial resistance genes and resistant bacteria are neither confined to animals and humans nor by geographic boundaries. This study principally investigated 
the predominance of ESBL-producing gram-negative isolates in retail food samples. We processed 436 food samples, including raw and processed foods. Among these samples, $352(80.73 \%)$ were culture-positive.

In total, 408 g-negative bacterial isolates were recovered from 352 positive cultures, of which $42.4 \%$ were ESBL producers. Our study indicated a $42.4 \%$ ESBL rate, which was higher than the $25.4 \%$ rate reported by Zurfluh et al. and lower than the $79.2 \%$ rate found by Richter et al. [21,22]. However, the ESBL proportion found in our study was higher than those reported in Vietnam [23] and Southwest Ethiopia [24], and lower than that reported in China [25]. The inconsistent prevalence rates may reflect differences in the study methodology, isolation technique, sample size and type, and collection process; thus, the comparison of results from different countries may not be feasible.

A high (51.5\%) incidence of ESBL producers in raw foods (vegetables, fruits, salads, juice, and milk) was detected in our study, which was in agreement with the results of previous reports from South Africa and Pakistan [3,22]. These findings indicate that raw and fresh foods can propagate and expand ESBL and drug resistance genes to consumers, as previously reported $[26,27]$. Another critical finding is that manure of animal origin, sewage and sludge, pesticides, and crop wastewater pollute soil and crops, providing a source of antibiotic resistance.

We also observed prevalent ESBL production in processed food (48.5\%), which indicates cross-contamination in the production process. Possible sources of contamination in processed food could include food handlers, food utensils, unsafe temperatures, and poor personal hygiene. A study in China reported more ESBL production in frozen food than in raw food, which is in contrast to our results [25].

The overwhelming majority of ESBL-producing bacterial isolates in our study were K. pneumoniae and E. coli, followed by S. typhimurium, E. cloacae, and P. aeruginosa, which was consistent with previous results in China and Vietnam [21,28]. Two additional studies reported K. pneumoniae, E. coli, S. typhimurium, E. cloacae, Citrobacter, and P. aeruginosa in a variety of food samples $[29,30]$. We found K. pneumoniae to be the predominant strain in both raw and processed foods, followed by E. coli. Two studies in China showed similar results, identifying E. coli as the most prevalent organism [28,31]. Interestingly, S. typhimurium was also found in raw $(14.41 \%)$ and processed foods $(5.91 \%)$, indicating poor hygiene and inadequate hand-washing practices, as this bacterium is spread from unwashed hands of infected food handlers via improper preparation and poor handling of foods, resulting in food contamination. Sofy et al. reported S. typhimurium as the second-most prevalent organism isolated from food samples [2].

The presence of E.coli and P.aeruginosa in processed food indicated that the food was either undercooked or subjected to post-cooking contamination, which may have occurred due to improper handling, prolonged storage, inappropriate chilling, or several other conditions. In comparison, the presence of S. typhimurium and S. marcescens in raw food may be acquired during processing as these bacteria are not naturally present in food items. Additionally, polluted water may be sprayed on the vegetables or vegetables may be grown on polluted soil with contaminated irrigation water. Local sanitary conditions may exist during the processing, post-production, transportation, storage, and retail of fresh vegetables [32,33].

Previous studies mainly indicated the presence of environmental bacteria harboring chromosome-mediated beta-lactamase resistance genes, but our study and other recent studies produced different results. A high prevalence of pathogenic bacteria such as Salmonella and some opportunistic bacteria, including E. coli, K. pneumoniae, Citrobacter spp. and Enterobacter spp., which not only cause community-acquired human infections but also carry plasmid-mediated resistance genes [34-36] was found.

Generally, $b l a_{\mathrm{TEM}}, b l a_{\mathrm{SHV}}$, and $b l a_{\mathrm{CTX}-\mathrm{M}}$ are common beta-lactamase genes found broadly. However, CTX-M- $\beta$-lactamase is the most prevalently reported ESBL worldwide $[37,38]$. Our study demonstrated the distributions of various ESBL genes, with bla $_{\text {CTX-M }}$ showing high prevalence, which is in agreement with previous results $[37,38]$. Ac- 
cording to PCR results, 83 (55\%) bla $a_{\mathrm{CTX}-\mathrm{M}}, 39$ (26\%) bla $a_{\mathrm{SHV}}$, and 28 (19\%) bla $a_{\mathrm{TEM}}$ genes were detected in our study, corresponding to previous findings [25]. We identified bla $a_{\mathrm{CTX}-\mathrm{M}-1}$ as the dominant variant among K. pneumoniae, E. cloacae, and E. coli, while bla ${ }_{\mathrm{CTX}-\mathrm{M}-15}$ was the most prevalent among E. coli and K. pneumoniae isolates in some previous reports [21,22].

High antimicrobial resistance against various groups of antibiotics was observed in our study, indicating a multidrug-resistance phenotype. Analysis of the antibiotic susceptibility profile revealed that most isolates were resistant to ampicillin (90\%), which is consistent with previous reporting in China [25]. We found high resistance to the cephalosporin antibiotic cefuroxime (51\%), and similar results were reported in a previous study [39]. For carbapenem, a moderate resistance rate was found, which is in agreement with previous reporting in China, indicating misuse of carbapenem drugs. This study revealed minimal resistance to tigecycline $(6 \%)$ and colistin $(2 \%)[13,40]$.

Heavy metals also exert antimicrobial effects, and some hospitals use metallic coppercoated surfaces to reduce the risk of nosocomial contamination [39]. Co-selection of antimicrobial and heavy metal resistance in bacteria has greatly affected the efficacy of available drugs and therapies for infectious diseases. To understand the role of heavy metals, we assessed all isolates against four heavy metals with increasing concentrations up to $1500 \mu \mathrm{g} / \mathrm{mL}$. The isolates indicated potential heavy metal contamination in RTE food samples. We found no clear differences between resistance patterns. However, selective screening of heavy metal-resistant isolates showed that those with maximal resistance were also ESBL producers. Many studies have reported the potential threat to the environment and foodstuff posed by heavy metals [41,42].

The presence of arsenic-, chromium-, molybdenum-, cobalt-, and mercury-resistant isolates in food samples can be attributed to the accumulation of high metal salt concentrations in soil and water, resulting in contamination with heavy metals such as arsenic, and the use of metal utensils. Metal resistance trends varied considerably depending on the sample site and sample type. There was a significant correlation between antibiotic resistance and metal resistance, indicating that these properties are linked. This study revealed high bacterial loads in foods readily and regularly consumed. The results established the incidence of ESBL-producing and heavy metal-resistant pathogenic bacteria in RTE foods sold locally in Punjab, Pakistan.

The present study highlighted several areas for improvement. The implementation of hygienic practices is mandatory throughout the food cycle, starting from manufacturing, processing, and cooking. Fruits and vegetables are irrigated with sewage in several fields in Pakistan. The use of contaminated water in washing the food imposes another risk of contamination. The use of contaminated hands and water in milking leads to milk contamination. Boiled or un-contaminated water should be used to water the vegetables and fruits. The implementation of vigilant hygienic measures on each of the steps mentioned above from the vendors and the consumers could help to reduce the risk of pathogen transmission. Effective surveillance and monitoring of food items and establishing and imposing regulations will ultimately reduce contaminants in the food samples.

To our knowledge, this is the first report of the high occurrence of coexisting ESBL production and heavy metal resistance in food samples in Pakistan which provides strength to this study; however, there is a limitation, too. We were unable to do molecular identification of metal resistant genes in these isolates, and it would be interesting to see the correlation of metal resistance genes and antibiotic-resistant genes in further studies.

\section{Conclusions}

Our results show that raw and processed foods, including vegetables, salad, fruits, and frozen meat, are important for disseminating ESBL genes, which pose significant consumer health risks. The highest incidence was reported for blaCTXM-1, followed by blaSHV-12, blaTEM-1, and blaCTXM-2. Strict policies and surveillance programs regarding the use of antimicrobial agents in the food and agriculture industry are urgently needed. This 
study also shows that heavy metal use provides a potent and previously underappreciated antibiotic resistance source.

Author Contributions: Conceptualization, K.J., H.E., K.O.A.A. and A.R.; methodology, I.A.; H.Y., S.Y.; software, K.J.; formal analysis, K.J., A.R., S.N.A.B., N.A. and A.E.A.; investigation, K.J., S.Y., K.O.A.A. and A.A.M.A.; resources, K.J., I.A. and H.E.; data curation, H.Y., A.R.; writing-original draft preparation I.A., S.Y., A.E.A., A.A.M.A.; writing-review and editing N.A., A.E.A., S.N.A.B.; supervision, K.J., A.R., H.Y. and project administration, K.J.; funding acquisition, K.J., H.E., S.Y. and A.E.A. All authors have read and agreed to the published version of the manuscript.

Funding: The authors extend their appreciation to the Deputyship for Research and Innovation, Ministry of Education in Saudi Arabia for funding this work through the grant number "375213500".

Institutional Review Board Statement: Not applicable.

Informed Consent Statement: Not applicable.

Data Availability Statement: Data are contained within the article.

Acknowledgments: The authors extend their appreciation to the Deputyship for Research and Innovation, Ministry of Education in Saudi Arabia, and the central laboratory at Jouf University for supporting this study.

Conflicts of Interest: The authors declare no conflict of interest.

\section{References}

1. Iseppi, R.; de Niederhäusern, S.; Bondi, M.; Messi, P.; Sabia, C. Extended-Spectrum $\beta$-Lactamase, AmpC, and MBL-Producing Gram-Negative Bacteria on Fresh Vegetables and Ready-to-Eat Salads Sold in Local Markets. Microb. Drug Resist. 2018, 24, 1156-1164. [CrossRef]

2. Sofy, A.R.; Sharaf, A.E.-M.M.; Al Karim, A.G.; Hmed, A.A.; Moharam, K.M. Prevalence of the harmful gram-negative bacteria in ready-to-eat foods in Egypt. Food Public Health 2017, 7, 59-68.

3. Samad, A.; Abbas, F.; Ahmad, Z.; Pokryrshko, O.; Asmat, T.M. Prevalence of foodborne pathogens in food items in Quetta, Pakistan. Pak. J. Zool. 2018, 50, 1-4. [CrossRef]

4. Dewey-Mattia, D.; Manikonda, K.; Hall, A.J.; Wise, M.E.; Crowe, S.J. Surveillance for Foodborne Disease Outbreaks-United States, 2009-2015. MMWR Surveill. Summ. 2018, 67, 1-11. [CrossRef]

5. Mesbah Zekar, F.; Granier, S.A.; Marault, M.; Yaici, L.; Gassilloud, B.; Manceau, C.; Touati, A.; Millemann, Y. From Farms to Markets: Gram-Negative Bacteria Resistant to Third-Generation Cephalosporins in Fruits and Vegetables in a Region of North Africa. Front. Microbiol. 2017, 8, 1569. [CrossRef] [PubMed]

6. Huizinga, P.; Schrauwen, E.; García-Cobos, S.; Willemsen, I.; Verhulst, C.; Friedrich, A.W.; Savelkoul, P.H.M.; Rossen, J.W.; Kluytmans, J. Extended-spectrum beta-lactamase producing Enterobacteriaceae (ESBL-E) isolated from bean sprouts in the Netherlands. PLoS ONE 2018, 13, e0203338. [CrossRef] [PubMed]

7. Blaak, H.; van Hoek, A.H.; Veenman, C.; Docters van Leeuwen, A.E.; Lynch, G.; van Overbeek, W.M.; de Roda Husman, A.M. Extended spectrum B-lactamase- and constitutively AmpC-producing Enterobacteriaceae on fresh produce and in the agricultural environment. Int. J. Food Microbiol. 2014, 168-169, 8-16. [CrossRef]

8. Lavilla, S.; González-López, J.J.; Miró, E.; Domínguez, A.; Llagostera, M.; Bartolomé, R.M.; Mirelis, B.; Navarro, F.; Prats, G. Dissemination of extended-spectrum beta-lactamase-producing bacteria: The food-borne outbreak lesson. J. Antimicrob. Chemother. 2008, 61, 1244-1251. [CrossRef] [PubMed]

9. Calbo, E.; Freixas, N.; Xercavins, M.; Riera, M.; Nicolás, C.; Monistrol, O.; Solé Mdel, M.; Sala, M.R.; Vila, J.; Garau, J. Foodborne nosocomial outbreak of SHV1 and CTX-M-15-producing Klebsiella pneumoniae: Epidemiology and control. Clin. Infect. Dis. 2011, 52, 743-749. [CrossRef]

10. Sanneh, B.; Kebbeh, A.; Jallow, H.S.; Camara, Y.; Mwamakamba, L.W.; Ceesay, I.F.; Barrow, E.; Sowe, F.O.; Sambou, S.M.; Baldeh, I.; et al. Prevalence and risk factors for faecal carriage of Extended Spectrum $\beta$-lactamase producing Enterobacteriaceae among food handlers in lower basic schools in West Coast Region of The Gambia. PLoS ONE 2018, 13, e0200894. [CrossRef]

11. Bondi, M.; Messi, P.; Halami, P.M.; Papadopoulou, C.; de Niederhausern, S. Emerging microbial concerns in food safety and new control measures. BioMed Res. Int. 2014, 2014, 251512. [CrossRef]

12. Yu, Z.; Gunn, L.; Wall, P.; Fanning, S. Antimicrobial resistance and its association with tolerance to heavy metals in agriculture production. Food Microbiol. 2017, 64, 23-32. [CrossRef]

13. Yang, Q.E.; Agouri, S.R.; Tyrrell, J.M.; Walsh, T.R. Heavy Metal Resistance Genes Are Associated with bla(NDM-1)- and bla(CTX-M-15)-Carrying Enterobacteriaceae. Antimicrob. Agents Chemother. 2018, 62. [CrossRef]

14. Younas, S.; Ejaz, H.; Zafar, A.; Ejaz, A.; Saleem, R.; Javed, H. AmpC beta-lactamases in Klebsiella pneumoniae: An emerging threat to the paediatric patients. J. Pak. Med. Assoc. 2018, 68, 893-897. 
15. Amin, H.; Zafar, A.; Ejaz, H.; Jameel, N.U. Phenotypic characterization of ESBL producing Enterobacter cloacae among children. Pak. J. Med. Sci. 2013, 29, 144-147. [CrossRef]

16. Clinical and Laboratory Standards Institute (CLSI). Performance Standards for Antimicrobial Susceptibility Testing; CLSI Supplement M100; Clinical and Laboratory Standard Institute (CLSI): Wayne, PA, USA, 2019.

17. Ejaz, H.; Ul-Haq, I.; Mahmood, S.; Zafar, A.; Mohsin Javed, M. Detection of extended-spectrum $\beta$-lactamases in Klebsiella pneumoniae: Comparison of phenotypic characterization methods. Pak. J. Med. Sci. 2013, 29, 768-772. [CrossRef] [PubMed]

18. Ejaz, H.; Younas, S.; Abosalif, K.O.A.; Junaid, K.; Alzahrani, B.; Alsrhani, A.; Abdalla, A.E.; Ullah, M.I.; Qamar, M.U.; Hamam, S.S.M. Molecular analysis of blaSHV, blaTEM, and blaCTX-M in extended-spectrum $\beta$-lactamase producing Enterobacteriaceae recovered from fecal specimens of animals. PLoS ONE 2021, 16, e0245126. [CrossRef] [PubMed]

19. Adekanmbi, A.O.; Falodun, O.I. Heavy metals and antibiotics susceptibility profiles of Staphylococcus aureus isolated from several points receiving daily input from the Bodija Abattoir in Ibadan, Oyo State, Nigeria. Adv. Microbiol. 2015, 5, 871. [CrossRef]

20. Dweba, C.C.; Zishiri, O.T.; El Zowalaty, M.E. Isolation and Molecular Identification of Virulence, Antimicrobial and Heavy Metal Resistance Genes in Livestock-Associated Methicillin-Resistant Staphylococcus aureus. Pathogens 2019, 8, 79. [CrossRef]

21. Zurfluh, K.; Nüesch-Inderbinen, M.; Morach, M.; Zihler Berner, A.; Hächler, H.; Stephan, R. Extended-spectrum- $\beta$-lactamaseproducing Enterobacteriaceae isolated from vegetables imported from the Dominican Republic, India, Thailand, and Vietnam. Appl. Environ. Microbiol. 2015, 81, 3115-3120. [CrossRef]

22. Richter, L.; Du Plessis, E.M.; Duvenage, S.; Korsten, L. Occurrence, Identification, and Antimicrobial Resistance Profiles of Extended-Spectrum and AmpC $\beta$-Lactamase-Producing Enterobacteriaceae from Fresh Vegetables Retailed in Gauteng Province, South Africa. Foodborne Pathog. Dis. 2019, 16, 421-427. [CrossRef]

23. Le, H.V.; Kawahara, R.; Khong, D.T.; Tran, H.T.; Nguyen, T.N.; Pham, K.N.; Jinnai, M.; Kumeda, Y.; Nakayama, T.; Ueda, S. Widespread dissemination of extended-spectrum $\beta$-lactamase-producing, multidrug-resistant Escherichia coli in livestock and fishery products in Vietnam. Int. J. Food Contam. 2015, 2, 1-6. [CrossRef]

24. Abayneh, M.; Tesfaw, G.; Woldemichael, K.; Yohannis, M.; Abdissa, A. Assessment of extended-spectrum $\beta$-lactamase (ESBLs)— Producing Escherichia coli from minced meat of cattle and swab samples and hygienic status of meat retailer shops in Jimma town, Southwest Ethiopia. BMC Infect. Dis. 2019, 19, 897. [CrossRef] [PubMed]

25. Ye, Q.; Wu, Q.; Zhang, S.; Zhang, J.; Yang, G.; Wang, J.; Xue, L.; Chen, M. Characterization of Extended-Spectrum $\beta$-LactamaseProducing Enterobacteriaceae From Retail Food in China. Front. Microbiol. 2018, 9, 1709. [CrossRef] [PubMed]

26. Reuland, E.A.; Al Naiemi, N.; Raadsen, S.A.; Savelkoul, P.H.; Kluytmans, J.A.; Vandenbroucke-Grauls, C.M. Prevalence of ESBL-producing Enterobacteriaceae in raw vegetables. Eur. J. Clin. Microbiol. Infect. Dis. 2014, 33, 1843-1846. [CrossRef] [PubMed]

27. Raphael, E.; Wong, L.K.; Riley, L.W. Extended-Spectrum Beta-lactamase gene sequences in gram-negative saprophytes on retail organic and nonorganic spinach. Appl. Environ. Microbiol. 2011, 77, 1601-1607. [CrossRef] [PubMed]

28. Ye, Q.; Wu, Q.; Zhang, S.; Zhang, J.; Yang, G.; Wang, H.; Huang, J.; Chen, M.; Xue, L.; Wang, J. Antibiotic-Resistant Extended Spectrum $B$-Lactamase- and Plasmid-Mediated AmpC-Producing Enterobacteriaceae Isolated from Retail Food Products and the Pearl River in Guangzhou, China. Front. Microbiol. 2017, 8, 96. [CrossRef]

29. Liu, S.; Kilonzo-Nthenge, A. Prevalence of Multidrug-Resistant Bacteria from U.S.-Grown and Imported Fresh Produce Retailed in Chain Supermarkets and Ethnic Stores of Davidson County, Tennessee. J. Food Prot. 2017, 80, 506-514. [CrossRef]

30. Falomir, M.P.; Gozalbo, D.; Rico, H. Coliform bacteria in fresh vegetables: From cultivated lands to consumers. In Current Research, Technology and Education Topics in Applied Microbiology and Microbial Biotechnology; Formatex Research Center: Badajoz, Spain, 2010; Volume 2, pp. 1175-1181.

31. Yang, J.F.; Ying, G.G.; Zhao, J.L.; Tao, R.; Su, H.C.; Liu, Y.S. Spatial and seasonal distribution of selected antibiotics in surface waters of the Pearl Rivers, China. J. Environ. Sci. Health B 2011, 46, 272-280. [CrossRef]

32. Mritunjay, S.K.; Kumar, V. A study on prevalence of microbial contamination on the surface of raw salad vegetables. 3 Biotech 2017, 7, 13. [CrossRef]

33. Mengistu, D.A.; Tolera, S.T. Prevalence of Microorganisms of Public Health Significance in Ready-to-Eat Foods Sold in Developing Countries: Systematic Review and Meta-Analysis. Int. J. Food Sci. 2020, 2020, 8867250. [CrossRef] [PubMed]

34. Jung, D.; Rubin, J.E. Identification of antimicrobial resistant bacteria from plant-based food products imported into Canada. Int. J. Food Microbiol. 2020, 319, 108509. [CrossRef] [PubMed]

35. Sapkota, S.; Adhikari, S.; Pandey, A.; Khadka, S.; Adhikari, M.; Kandel, H.; Pathak, S.; Pandey, A. Multi-Drug resistant extendedspectrum beta-lactamase producing E. coli and Salmonella on raw vegetable salads served at hotels and restaurants in Bharatpur, Nepal. BMC Res. Notes 2019, 12, 516. [CrossRef] [PubMed]

36. Van Hoek, A.H.; Veenman, C.; van Overbeek, W.M.; Lynch, G.; de Roda Husman, A.M.; Blaak, H. Prevalence and characterization of ESBL- and AmpC-producing Enterobacteriaceae on retail vegetables. Int. J. Food Microbiol. 2015, 204, 1-8. [CrossRef] [PubMed]

37. Ben-Ami, R.; Rodríguez-Baño, J.; Arslan, H.; Pitout, J.D.; Quentin, C.; Calbo, E.S.; Azap, O.K.; Arpin, C.; Pascual, A.; Livermore, D.M.; et al. A multinational survey of risk factors for infection with extended-spectrum beta-lactamase-producing enterobacteriaceae in nonhospitalized patients. Clin. Infect. Dis. 2009, 49, 682-690. [CrossRef] [PubMed]

38. Peirano, G.; Sang, J.H.; Pitondo-Silva, A.; Laupland, K.B.; Pitout, J.D. Molecular epidemiology of extended-spectrum- $\beta$-lactamaseproducing Klebsiella pneumoniae over a 10 year period in Calgary, Canada. J. Antimicrob. Chemother. 2012, 67, 1114-1120. [CrossRef] [PubMed] 
39. Grass, G.; Rensing, C.; Solioz, M. Metallic copper as an antimicrobial surface. Appl. Environ. Microbiol. 2011, 77, $1541-1547$. [CrossRef]

40. Chen, J.; Jin, M.; Qiu, Z.G.; Guo, C.; Chen, Z.L.; Shen, Z.Q.; Wang, X.W.; Li, J.W. A survey of drug resistance bla genes originating from synthetic plasmid vectors in six Chinese rivers. Environ. Sci. Technol. 2012, 46, 13448-13454. [CrossRef]

41. Bvenura, C.; Afolayan, A.J. Heavy metal contamination of vegetables cultivated in home gardens in the Eastern Cape. S. Afr. J. Sci. 2012, 108, 1-6. [CrossRef]

42. Iwegbue, C.; Nwajei, G.; Iyoha, E. Heavy metal residues of chicken meat and gizzard and turkey meat consumed in southern Nigeria. Bulg. J. Vet. Med. 2008, 11, 275-280. 